\title{
Carbomer Viscosupplementation in the Equine Middle Carpal Joint JM Rosser*
}

Clinic for Equine Surgery, Veterinary Medical University, Austria

\begin{abstract}
Joint visco supplementation is widely used in the sport horse industry, commonly utilizing hyaluronan. Similar to hyaluronan, the carbomer molecule has bioadhesive and viscoelastic properties, and has been 15 studied for use in human medicine. Carbomers are generally inert biologically, non-irritating, and have been used in humans as injectable depots for time-release medications. Our objective was to describe the outcome of intra-articular viscosupplementation with carbomer in four horses. Four healthy adult mares participated in this study. Baseline lameness examinations, aseptic arthrocenteses and synovial fluid analyses were performed. Three $\mathrm{mL}$ of $3 \%$ carbomer was then 20 injected into each left middle carpal joint. Lameness evaluations were repeated daily for each horse for ten days post-injection or until the time of euthanasia. At euthanasia, synovial fluid analyses were repeated on the left middle carpal joint. Samples of synovium from the left middle carpal joints were submitted for histopathology. Articular cartilage of the third carpal bone including subchondral bone was submitted for histology and confocal microscopy. 25 Carbomer viscosupplementation of the middle carpal joint caused severe lameness in $3 / 4$ mares. The fourth mare developed moderate lameness after injection. Confocal microscopy was negative for cartilaginous defects. Histology of all treated samples revealed varying degrees of small cell inflammatory infiltrate into the subsynovia. With no abnormal findings in samples of cartilage or subchondral bone, lameness is attributable to 30 severe synovitis, which is consistent with histologic findings of synovium in all cases. The current formulation of carbomer is therefore not suitable for intra-articular viscosupplementation in horses.
\end{abstract}

Keywords: Carpal Joint; Subchondral bone; Viscosupplementation

\section{Introduction}

Osteoarthritis has been shown to have a significant economic impact on the sport horse industry [1]. It is the most common type of arthritis and is associated with both normal and abnormal wear and tear on a joint [2]. Osteoarthritis is the clinical manifestation of progressive cartilaginous lesions and is a chronic, progressive condition in horses which can ultimately lead to fragmentation of cartilage or bone and is typically associated with decreased viscosity of joint fluid, synovial inflammation and often lameness.

Treatment of osteoarthritis is generally initiated due to signs of lameness. Frequently, horses will be temporarily rested with minimal to no improvement of the lameness. Non-steroidal antiinflammatory drugs are often administered via various routes as needed symptomatically by the individual, and can be very useful for horses with mild osteoarthritis [2,3]. Polysulfated polysaccharides have been administered systemically and intra-articularly for osteoarthritis resulting in significant clinical improvement ${ }^{3}$. Other intra-articular medications have also been used to treat osteoarthritis, including steroid administration, hyaluronic acid injections, and immune modulatory autologous interleukin 1 receptor antagonist protein [3].

Intra-articular medications have two aims, the first being reduction in pain and the second halting progression of cartilage degeneration [3]. Corticosteroids achieve these results via innate antiinflammatory affects. However, intra-articular hyaluronan is not only anti-inflammatory [4], but also increases the viscosity and elasticity of synovial fluid locally, thereby enhancing endogenous hyaluronan [5]. This procedure is often referred to as joint viscosupplementation [6].

Joint viscosupplementation has been used in veterinary medicine since the 1970's, particularly in the sport horse industry [6]. Most commonly, joint viscosupplementation utilises a preparation of hyaluronan, which is available in a wide array of molecular weight $[2,7,8]$. Although hyaluronan possesses a relatively short half-life within synovial structures [9], the local viscoelastic and anti-inflammatory effects can last several months. Hyaluronan is a structural component of both synovial fluid and articular cartilage. Injectable supplementation of hyaluronan thereby increases the viscosity of the synovial fluid, acting as a lubricant, metabolic medium, and shock absorber [10-12]. It is important to note, however, that intra-articular administration of hyaluronan does not stimulate endogenous hyaluronan synthesis of increased molecular weight, nor does it inhibit synthesis of collagenase [13]. Additionally, hyaluronan dictates fluid outflow from the synovial compartment by creating flow-dependent concentration polarization $[12,14,15]$.

Carbomer is a polyacrylic acid polymer available in a variety of molecular weights and can exist in linear, branched or cross-linked configurations $[16,17]$. When suspended in water, the acidic carboxyl groups partially dissociate, resulting in the production of a flexible coil. This is because the carbomer polymer, though hydrophilic, is not water soluble. Due to the addition of alkaline compounds, salt formation and subsequent repulsion between anions cause the formula to gel $[18,19]$. The carbomer molecule has inherent bioadhesive, viscoelastic, and rheologic properties, and has been studied extensively for use in ophthalmology [20] and reconstructive surgery in human medicine. Additionally, carbomers are generally inert biologically and nonirritating [21,22].

Current applications for carbomer use abound in human medicine. Different preparations of carbomers have been used in ophthalmology for maintenance of mucoid tear film layer [23]. Carbomer has also been used in plastic surgery and dermatology as a filling agent for cosmetic purposes $[16,17,21,22]$. Additionally, carbomer has been transmucosally

*Corresponding author: JM Rosser, Clinic for Equine Surgery, Veterinary Medical University, Austria, Tel: +43664602576646; E-mail: jmrosser58@gmail.com

Received December 09, 2013; Accepted February 14, 2014; Published February 17,2014

Citation: Rosser JM (2014) Carbomer Viscosupplementation in the Equine Middle Carpal Joint. J Veterinar Sci Technol 5: 158. doi:10.4172/2157-7579.1000158

Copyright: @ 2014 Rosser JM. This is an open-access article distributed under the terms of the Creative Commons Attribution License, which permits unrestricted use, distribution, and reproduction in any medium, provided the original author and source are credited. 
injected into hypoplastic arytenoids and vocal cords in order to aid in normal airway abduction and vocal function. Carbomers have also been used pharmaceutically as depots to release corticosteroids within a tissue over long periods of time $[18,19,24,25]$.

We hypothesised that intra-articular carbomer use would have no significant negative consequences when tried experimentally in the middle carpal joints of horses and that synovial fluid analysis, articular cartilage histopathology, and subchondral bone confocal microscopy would display no subsequent adverse effects. Secondly, we hypothesised that carbomer gel would be retained within the joint capsule after injection. Finally, we hypothesised that intra-articular administration of carbomer gel would improve the osteoarthritic status of the clinically or experimentally affected joint. This pilot study tested only the first two hypotheses.

\section{Materials and Methods}

We utilised a $3 \%$ concentration of nonbiodegradable carbomer 974P NF (carbomer gel, estimated molecular weight $7 \times 10^{5}$ to $4 \times 10^{9}$ ), synthesised in ethyl acetate in order to decrease the residual solvent in the final product, as opposed to benzene dependent synthesis [23]. This method of synthesis achieves a cleaner product with less tissue reactivity. Carbomer was manufactured by an independent, off-site laboratory.

Four adult mares donated to the UW-VMTH Large Animal Surgery Service for euthanasia were selected to participate in the viscosupplementation pilot study. The animal use protocol for the viscosupplementation pilot study was approved by the University of Wisconsin Madison Research Animal Resources Center.

Baseline physical and lameness examinations were performed on each horse at admission and daily post injection. After aseptic preparation, arthrocentesis and subsequent synovial fluid analysis of the left middle carpal joint was performed and $3 \mathrm{~mL}$ of $3 \%$ carbomer gel injected. Two of the horses also had arthrocentesis of the right middle carpal joint at time zero for control purposes. One of these horses was injected with $0.9 \% \mathrm{NaCl}$ into the right middle carpal joint at time zero as a control. Finally, the fourth horse was injected subcutaneously with carbomer gel in the right caudal cervical region at time zero in order to utilise non synovial and non-articular tissue for control purposes.

Physical exams were repeated twice daily for each horse post injection until the time of euthanasia, and lameness evaluations were repeated daily.

Horses in the study were euthanatised 10 days post injection unless lameness was considered to be so severe that continuing the study was inhumane to the individual.

At euthanasia, synovial fluid analysis was again performed on the left middle carpal joint as well as the right middle carpal joint in the two horses previously mentioned. Samples of synovium from the left middle carpal joints were collected and submitted for histopathology. The proximodorsal margin of articular cartilage of the third carpal bone including subchondral bone was removed using an osteotome and submitted for histology and confocal laser microscopy as follows: The $1.5 \mathrm{~mm}$ thick cartilage slices were stained by incubation in a 1.0 $\mathrm{ml}$ PBS containing $0.4 \mathrm{ml}$ calcein acetoxymethylester) $/ 10 \mathrm{ml}$ ethidium homodimer (Molecular Probes, Eugene, OR) for 30 minutes at room temperature. The method of determining the location of surviving cells was based on the knowledge that viable and nonviable cells differ in their ability to exclude fluorescent dyes. The cell membranes of dead, damaged or dying cells are penetrated by ethidium homodimer to stain their nuclei red. Living cells with intact plasma membranes and active esterases metabolize calcein (acetoxymethyl ester) and show cytoplasmic green fluorescence. The method of analysis utilized a 1.5 $\mathrm{mm}$ thick cartilage slice that was placed on a glass slide and moistened by several drops of PBS. A confocal laser microscope (MRC-1024, BioRad, Hemel Hempsted/Cambridge, England) equipped with a krypton/ argon laser and the necessary filter systems (fluorecein: 522DF32 and rhodamine: 585EFLP) was employed using the triple-labeling technique as previously described. All cartilage samples were examined blinded to group assignment. The confocal laser microscope was calibrated using a micrometer measured through the objective lens $(2 \mathrm{x})$ used for this project. The pixel length measured on images was converted to micrometers. The mean depth of chondrocyte death (mean depth of 3 deepest areas of chondrocyte death) was determined in each CLM image of the osteochondral section with Adobe PhotoShop ${ }^{\text {тx }}$ (Adobe PhotoShop, Version 5.0.2, San Jose, CA).

\section{Results}

Signalment and synovial fluid analysis results for all four study horses are presented in Table 1 . No remaining carbomer gel was grossly apparent within the joint capsule at the time of sample harvest. No abnormalities were detected in any articular cartilage or subchondral bone specimens submitted for histopathology or confocal laser microscopy.

\section{Horse one}

Horse one presented with a history of recurrent lymphangitis and bilateral distal forelimb pitting oedema at presentation. The mare was grade III/V lame (AAEP Lameness Grading Scale) in the left forelimb upon presentation. As early as day 2 post injection, horse one began to display left middle carpal joint effusion and an increase in distal limb oedema of the left forelimb. Three days after intra articular injection, horse one's left middle carpal joint effusion was still grossly evident and distal limb oedema of the right forelimb was seen. Five days after injection, the left forelimb lameness had worsened to grade IV/V (after AAEP). By day seven, the mare was intermittently non weight bearing in the stall and consistently lame in the left forelimb at the walk. Topical application of DMSO and nitrofurazone in a sweat wrap technique was used for both forelimbs. Oral phenylbutazone $(2.2 \mathrm{mg} /$ $\mathrm{kg}$ ) was administered due to the severity of lameness. The mare was euthanatised nine days post injection due to severity of lameness and lack of response to rescue treatment.

At post mortem, horse one had a total protein of $6.0 \mathrm{~g} / \mathrm{dL}$ in the left middle carpal joint although total nucleated cell count was within normal limits (Table 1). Histology of the left middle carpal synovium

\begin{tabular}{|l|c|c|c|c|}
\hline Signalment & Horse 1 & Horse 2 & Horse 3 & Horse 4 \\
\hline Baseline Synovial TNCC: & $7 y 0$ TB mare & $9.5 y o$ App mare & $32 y 0$ Arab mare & $23 y o$ QHx mare \\
\hline Baseline Synovial TP: & $<2.5 \mathrm{~g} / \mathrm{dL}$ & $<2.5 \mathrm{~g} / \mathrm{dL}$ & $<2.5 \mathrm{~g} / \mathrm{dL}$ & $<2.5 \mathrm{~g} / \mathrm{dL}$ \\
\hline Euthanasia at day: & 9 & 4 & 5 & 8 \\
\hline L carpus synov TNCC & 720 & 18960 & 940 & 760 \\
\hline L carpus synov TP & $6.0 \mathrm{~g} / \mathrm{dL}$ & $5.4 \mathrm{~g} / \mathrm{dL}$ & $4.0 \mathrm{~g} / \mathrm{dL}$ & $3.3 \mathrm{~g} / \mathrm{dL}$ \\
\hline R carpus synov TNCC & 160 & 140 & $\mathrm{n} / \mathrm{a}$ & $\mathrm{n} / \mathrm{a}$ \\
\hline R carpus synov TP & $<2.5 \mathrm{~g} / \mathrm{dL}$ & $<2.5 \mathrm{~g} / \mathrm{dL}$ & $\mathrm{n} / \mathrm{a}$ & $\mathrm{n} / \mathrm{a}$ \\
\hline
\end{tabular}

Synoviocentesis results for all horses pre- and post-injection, including controls (Right middle carpal joint of 2 horses). TNCC: total nucleated cell count. TP: total protein. $\mathrm{L}$ carpus: left middle carpal joint. $\mathrm{R}$ carpus: right middle carpal joint. TB: thoroughbred. App: appaloosa. Arab: Arabian. QHx: quarter horse cross. G/dL: grams per deci-liter.

Table 1: Laboratory results of each animal's synoviocentesis and the study day on which the animal was euthanatised. 
Citation: Rosser JM (2014) Carbomer Viscosupplementation in the Equine Middle Carpal Joint. J Veterinar Sci Technol 5: 158. doi:10.4172/21577579.1000158

Page 3 of 6

revealed small cell inflammatory infiltrate and detachment of the synovial intima when compared to the right middle carpal synovium as a control (Figure 1). Histology of cartilage and subchondral bone of the left and right third carpal bones were within normal limits. Confocal microscopy of cartilage from the left and right third carpal bones was unremarkable (Figure 2). Aseptic synoviocentesis of the left
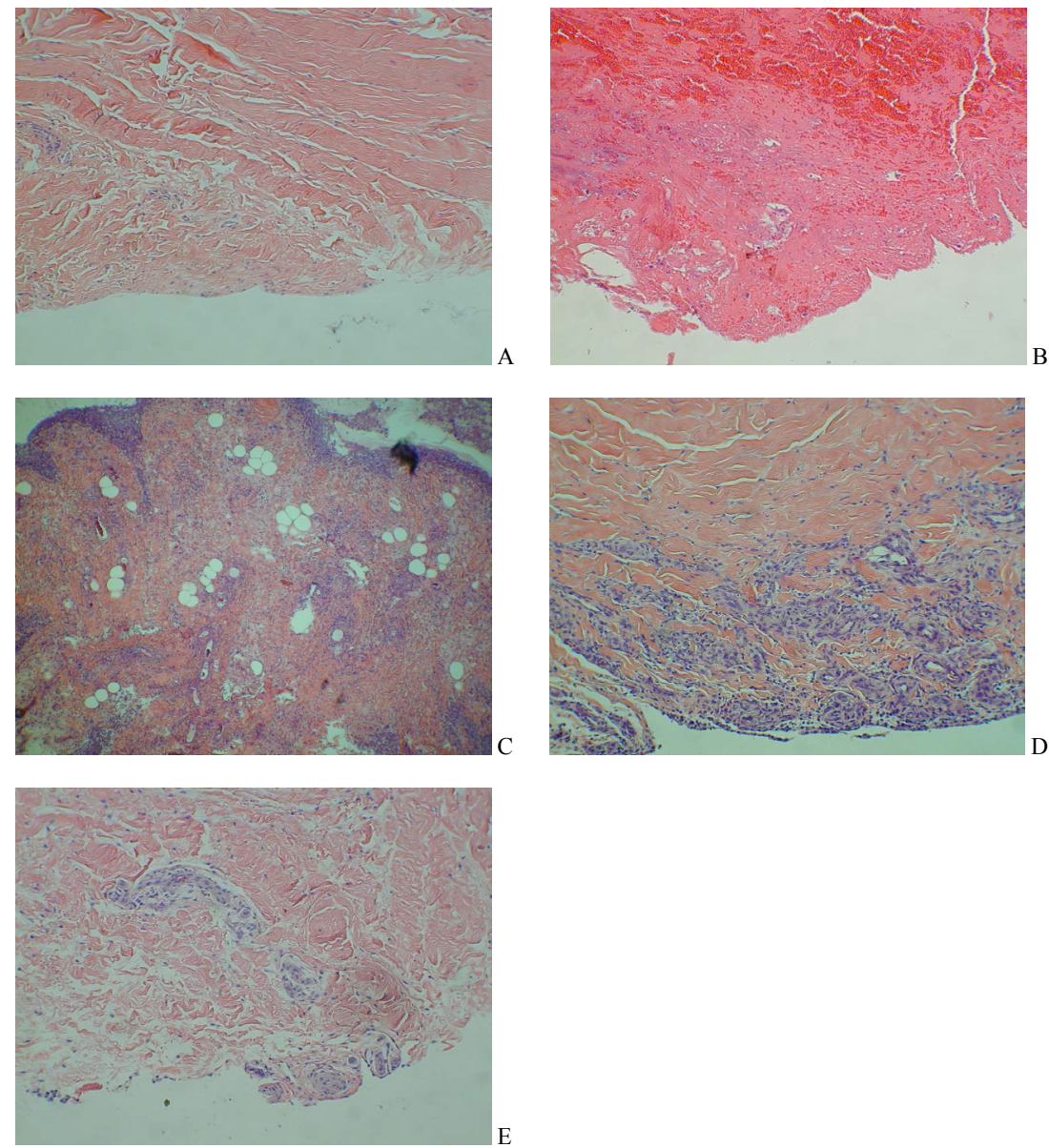

Figure 1: Histology slides of middle carpal joint synovium taken at post mortem evaluation of each study horse. All images were stained with H\&E and taken at $40 \mathrm{x}$ magnification. A) Horse 1, control sample (right middle carpal synovium). B) Horse 1, treated sample (left middle carpal synovium). Note the obvious presence of red blood cells, lack of collagen fibre orientation, and small cell infiltrate within the fibrolayer of the synovium. The subsynovial layer appears to be detached or absent, most likely consistent with handling artifact. C) Horse 2, treated sample (left middle carpal synovium). Note increased cellularity in this sample as compared to A however, in this animal's self-controlled sample (right middle carpal synovium), mild hypercellularity was also present. D) Horse 3, treated sample (left middle carpal synovium). Note the inflammatory infiltrate seen in the subsynovia. E) Horse 4, treated sample (left middle carpal synovium). Note the small amount of inflammatory cell infiltrate accompanied by mild disorganisation of collagen fibres.

A) Confocal image of left carpus (treated) of horse 1

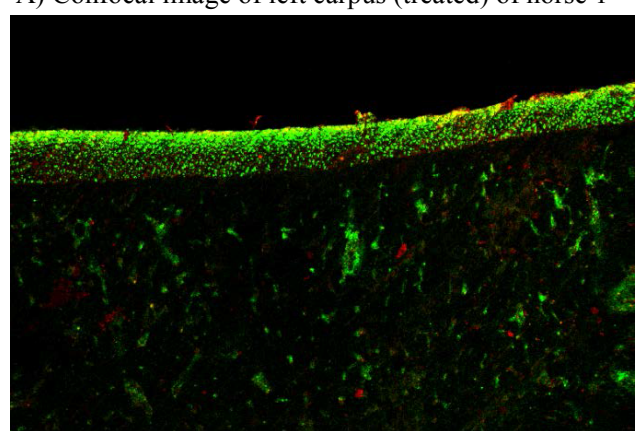

B) Confocal image of right carpus (control) of horse 1

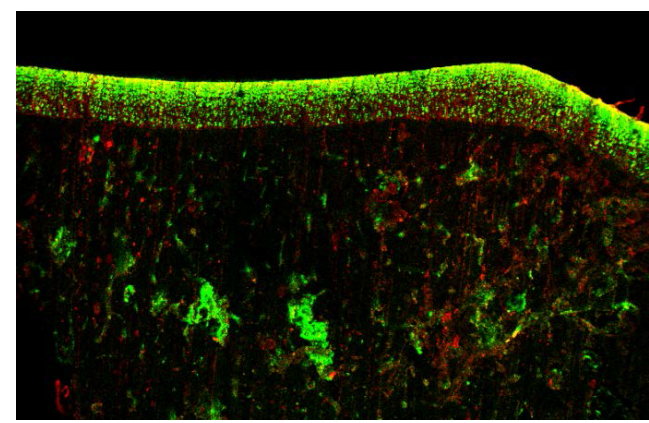

Figure 2: Confocal microscopy of Horse 1. Figure1A is the treated, left proximal third carpal bone sample including articular cartilage and subchondral bone within the left middle carpal joint. Figure $1 \mathrm{~B}$ is the control, right proximal third carpal bone sample. No appreciable differences were present between treated and control samples on confocal microscopy. 
middle carpal joint was submitted for aerobic and anaerobic culture and sensitivity with no resultant growth.

\section{Horse two}

Horse two presented with a historical diagnosis of pain in the palmar aspect of the foot and grade II/V (AAEP Lameness Grading Scale) bilateral forelimb lameness. By Day 2 post injection, the mare was febrile with a temperature of $102.8 \mathrm{~F}$ and lameness of the left forelimb had worsened to grade III/V (after AAEP). Effusion of the left middle carpal joint was grossly apparent. Three days post injection, horse two showed progressive lameness (grade IV/V, AAEP Lameness Grading Scale) as well as persistent left middle carpal joint effusion. Flunixin meglimine $(1.1 \mathrm{mg} / \mathrm{kg})$ was administered intravenously due to the severity of lameness. The mare was euthanatised four days post injection due to the severity of lameness and lack of response to rescue treatment.

At post mortem, horse two had a total protein of $5.4 \mathrm{~g} / \mathrm{dL}$ in the left middle carpal joint and a total nucleated cell count of 18960 (Table 1). Histology of the left middle carpal synovium revealed hypercellular infiltrate. Histology of cartilage and subchondral bone of the left and right third carpal bones was unremarkable (Figure 2). Synoviocentesis and fluid analysis of the right middle carpal joint at post mortem were within normal limits (Table 1). Right middle carpal synovial histology revealed increased cellularity. Right middle carpal cartilage and subchondral bone histology were unremarkable.

\section{Horse three}

Horse three presented with severe left carpal varus but was sound on baseline lameness evaluation. Twelve hours post injection the mare was non weight bearing lame in the left forelimb with effusion of the left middle carpal joint. Intramuscular butorphanol was administered for pain control. By day two, the left forelimb lameness had improved to grade III/V and diffuse oedema was present around the entire left carpus. On day three, the mare's lameness had again worsened to grade $\mathrm{IV} / \mathrm{V}$ on the left forelimb with periods of intermittent non weight bearing. Due to lack of improvement of clinical signs over the course of the following day, the mare was euthanatised for humane reasons on day five.

At post mortem, horse three had a total protein of $4.0 \mathrm{~g} / \mathrm{dL}$ in the left middle carpal joint and a total nucleated cell count of 940 (Table 1). Histology of the left middle carpal synovium revealed inflammatory infiltrate within the subsynovia and perivascular space, as well as thickened, inflamed synovial intima (Figure 2). Differential counts of the left middle carpal synovial fluid analysis were indicative of an inflammatory process. Cartilage histology, subchondral bone histology, and synovial fluid analysis of the right middle carpal joint were not performed in horse three.

\section{Horse four}

Horse four presented with a history of chronic refractory uveitis. Upon presentation, a perforating corneal ulcer was seen in the left eye, as well as a cataract rendering the left eye avisual. Due to the pain associated with corneal perforation, she was placed on 1 gram phenylbutazone orally every 12 hours for the duration of the study. Prior to phenylbutazone administration, the mare was sound on baseline lameness evaluation. One day post injection the mare displayed grade III/V left forelimb lameness (AAEP Lameness Grading Scale). On Day two, the horse exhibited left middle carpal effusion. On days four and five post injection, the left forelimb lameness fluctuated slightly between grade II and III/V, but on day five the mare was positive to left carpal flexion and the effusion of the left middle carpal joint was increased. Days six and seven post injection the mare continued to show a fluctuation between grade II and III/V in her left forelimb lameness. She was euthanatised on day 8 for humane purposes.

At post mortem, horse three had a total protein of $3.3 \mathrm{~g} / \mathrm{dL}$ in the left middle carpal joint and a total nucleated cell count of 760 (Table 1). Histology of the left middle carpal synovium revealed villous loss, with blunting of remaining villi and lymphoplasmacytic infiltrate (Figure 2). Cartilage histology, subchondral bone histology, and synovial fluid analysis of the right middle carpal joint were not performed in horse four.

\section{Discussion}

The severe cases of lameness post injection were unexpected, as were the high protein values within the synovial fluid analysis. If these reactions were due to a septic process, we would have expected to see higher total cell counts via arthrocentesis. Additionally, the left middle carpal joint of the first horse was aseptically sampled at euthanasia for culture as well as synovial fluid analysis, with negative culture results. Finally, one gas sterilized syringe of $3 \mathrm{~mL}$ carbomer gel was also cultured with no subsequent growth. However, no endotoxin assays were performed at our laboratory. It is possible that the synovium could be extremely sensitive to any endotoxin contamination, a condition which has been seen in administration of injectable ophthalmic preparations. If endotoxin were present in the carbomer gel formulation, it would have to be at very low levels, as general assays were run during manufacture. Another potential explanation for the severe arthritis is joint flare, as an acute aseptic synovitis, which would be more consistent with the high synovial fluid proteins in the absence of extremely elevated synovial fluid total nucleated cell counts seen in this pilot study.

Aseptic synovitis cases have been reported in horses. Acute inflammation post joint injection can be seen and is often difficult to differentiate from septic arthritis. Total nucleated cell counts in horses with aseptic synovitis are often elevated but rarely exceed $30,000 \mathrm{~g} / \mathrm{dL}$, whereas infectious arthritis frequently has an even more increased value. Both aseptic and septic synovitis can display increases in synovial fluid total protein. Obtaining a culture from an aseptically prepared arthrocentesis can be helpful in determining whether or not the joint was contaminated, but synovial cultures are commonly unable to identify causative bacteria or other infectious agents. Cytology of synovial fluid may also reveal degenerate neutrophils and/or free or intracellular bacteria, thereby aiding significantly in differentiating septic from aseptic arthritis. Interestingly, reports of eosinophilic or lymphocytic synovitis exist in the literature [26,27]. While our findings are not consistent with eosinophilic synovitis, it is possible that carbomer gel causes lymphocytic synovitis. Greater numbers would be required to prove this theory, but due to the severe lameness initiated by this treatment protocol, additional studies cannot be recommended without first definitively identifying the etiology of the resultant lamenesses seen in this experiment.

We were unable to ascertain objectively if the severity of lameness seen in our cases was directly affected by their initial lameness status.

Additionally, we expected that the carbomer gel would be grossly apparent within the joint capsule upon post-euthanasia arthrotomy. Although the gel was not biologically marked, we expected due to its high molecular weight and size that it would remain grossly apparent within the joint. 
Viscosity of the synovial fluid is difficult to assess due to inconsistency between laboratories and general lack of available information. Therefore, we were unable to include data regarding viscosity prior or subsequent to injection which afforded us no useful comparison.

Nonbiodegradable carbomers have been previously associated with granulomatous and foreign body reactions when injected subcutaneously in humans $[28,29]$. The nonbiodegradable nature of these substances prolongs the aesthetic filling effect but also increases the risk of infection or immune reaction. When used in plastic surgery and dermatologic procedures, lesions are typically attributed to inadequate injection technique or use of excess medication. Additionally, 3\% of cases reported by Lemperle et al. were seen to experience migration of the carbomer from the original injection site when used for breast augmentation. Granulomatous reactions have also been reported subsequent to use of biodegradable carbomers, which can spontaneously resolve, presumably due to the transient nature of hyaluronan used for soft tissue filler.

When used aesthetically in human subcutaneous tissues, polyacrylamide gel acts as filler via volumetric effect instead of relying on the host immune response for filling effect. Transient tissue reaction does occur and results in mild fibrosis with connective tissue fibers and vascularisation that act to tether the gel in place.

Another potential application of carbomer gels is the controlled delivery of bioactive agents [25,30]. Use of polymeric drug systems are currently popular in human medicine, but have limited employment in the realm of veterinary medicine at this time [31]. Clearly, this formulation will not be suitable for depot medication in equine synovial structures in its current state.

\section{Acknowledgements}

The authors would like to express their sincere gratitude for the assistance in study design, manuscript preparation, and technical assistance provided by $\mathrm{Dr}$. Alberto Serena, DVM and Dr. Peter Muir, PhD, DAC.

\section{References}

1. Goodrich LR, Nixon AJ (2006) Medical treatment of osteoarthritis in the horse - a review. Vet J 171: 51-69.

2. Frisbie DD (2005): Synovial Joint Biology and Pathobiology. In Auer J, Stick J (eds): Equine Surgery (ed 3). St. Louis, MO, Saunders 1036-1054.

3. Frisbie DD (2006) Principles of Treatment of Joint Disease, In Auer J, Stick J (eds): Equine Surgery (ed 3) St. Louis, MO, Saunders, Pp 1055-1073.

4. Strauss E, Schachter A, Frenkel S, Rosen J (2009) The efficacy of intraarticular hyaluronan injection after the microfracture technique for the treatment of articular cartilage lesions. Am J Sports Med 37: 720-726.

5. Frisbie DD, Kawcak CE, Mcllwraith CW, Werpy NM (2009) Evaluation of polysulfated glycosaminoglycan or sodium hyaluronan administered intraarticularly for treatment of horses with experimentally induced osteoarthritis. Am J Vet Res 70: 203-209.

6. Marshall KW (2000) Intra-articular hyaluronan therapy. Curr Opin Rheumato 12: $468-474$

7. Kotevoglu N, lyibozkurt PC, Hiz O, Toktas H, Kuran B (2006) A prospective randomised controlled clinical trial comparing the efficacy of different molecula weight hyaluronan solutions in the treatment of knee osteoarthritis. Rheumato Int 26: 325-330.

8. Gomis A, Pawlak M, Balazs EA, Schmidt RF, Belmonte C (2004) Effects of different molecular weight elastoviscoushyaluronan solutions on articular nociceptive afferents. Arthritis Rheum 50: 314-326.

9. Strauss EJ, Hart JA, Miller MD, Altman RD, Rosen JE (2009) Hyaluronic acid viscosupplementation and osteoarthritis: current uses and future directions. Am J Sports Med 37: 1636-1644.
10. Popot MA, Bonnaire Y, Guechot J, Toutain PL (2004)Hyaluronan in horses: physiological production rate, plasma and synovial fluid concentrations in control conditions and following sodium hyaluronate administration. Equine Vet Journ 36: 482-487.

11. Labens R, Mellor DJ, Voûte LC (2007) Retrospective study of the effect of intraarticular treatment of osteoarthritis of the distal tarsal joints in 51 horses. Ve Rec 161: 611-616.

12. Sabaratnam S, Arunan V, Coleman PJ, Mason RM, Levick JR (2005) Size selectivity of hyaluronan molecular sieving by extracellular matrix in rabbit synovial joints. J Physiol 567: 569-581.

13. Lynch TM, Caron JP, Arnoczky SP, Lloyd JW, Stick JA, et al. (1998) Influence of exogenous hyaluronan on synthesis of hyaluronan and collagenase by equine synoviocytes. Am J Vet Res 59: 888-892.

14. Scott D, Coleman PJ, Mason RM, Levick JR (2000) Action of polysaccharides of similar average mass but differing molecular volume and charge on fluid drainage through synovial interstitium in rabbit knees. J Physiol 528: 609-618.

15. Sabaratnam S, Mason RM, Levick JR (2004) Filtration rate dependence of hyaluronan reflection by joint-to-lymph barrier: evidence for concentration polarisation. J Physiol 557: 909-922.

16. Von Buselow S, Pallua N (2006) Efficacy and Safety of Polyacrylamide Hydrogel for Facial Soft-Tissue Augmentation in a 2 Year Follow-Up: A Prospective Multicenter Study for Evaluation of Safety and Aesthetic Results in 101 Patients. Plastic and Reconstructive Surgery September Supplement. 118: 85-91.

17. Wolters M, Lampe H (2009) Prospective multicenter study for evaluation of safety, efficacy, and esthetic results of cross-linked polyacrylamide hydrogel in 81 patients. Dermatol Surg 35: 338-343.

18. Islam MT, Rodríguez-Hornedo N, Ciotti S, Ackermann C (2004) Rheological characterization of topical carbomer gels neutralized to different $\mathrm{pH}$. Pharm Res 21: 1192-1199.

19. Tanna S, Sahota T, Clark J, Taylor MJ (2002) A covalently stabilised glucose responsive gel formulation with a Carbopol carrier. J Drug Target 10: 411-418.

20. Kaufman PL, Lütjen-Drecoll E, Hubbard WC, Erickson KA (1994) Obstruction of aqueous humor outflow by cross-linked polyacrylamide microgels in bovine, monkey, and human eyes. Ophthalmology 101: 1672-1679.

21. Christensen L, Breiting V, Janssen M, Vuust J, Hogdall E (2005) Adverse reactions to injectable soft tissue permanent fillers. Aesthetic Plast Surg 29 $34-48$

22. vonBuelow S, von Heimburg D, Pallua N (2005) Efficacy and safety of polyacrylamide hydrogel for facial soft-tissue augmentation. Plast Reconstr Surg 116: 1137-1146.

23. Ceulemans J, Ludwig A (2002) Optimisation of carbomer viscous eye drops: an in vitro experimental design approach using rheological techniques. Eur $\mathrm{J}$ Pharm Biopharm 54: 41-50.

24. Parojcić J, Ethurić Z, Jovanović M, Ibrić S, Jovanović D (2004) Influence of dissolution media composition on drug release and in-vitro/in-vivo correlation for paracetamol matrix tablets prepared with novel carbomer polymers. J Pharm Pharmacol 56: 735-741.

25. Young CR, Dietzsch C, Cerea M, Farrell T, Fegely KA, et al. (2005) Physicochemical characterization and mechanisms of release of theophylline from melt-extruded dosage forms based on a methacrylic acid copolymer. Int J Pharm 301: 112-120.

26. Climent F, Carmona JU, Cuenca R, Prades M (2007) Eosinophilicsynovitis of the tarsocrural joint in a horse. Vet Comp Orthop Traumatol 20: 142-145.

27. Burgess EC, Gillette D, Pickett JP (1986) Arthritis and panuveitis as manifestations of Borreliaburgdorferi infection in a Wisconsin pony. J Am Vet Med Assoc 189: 1340-1342.

28. Kawamura JY, Domaneschi C, Migliari DA, Sousa SO (2006) Foreign body reaction due to skin filler: a case report. Oral Surg Oral Med Oral Pathol Oral Radiol Endod 101: 469-471.

29. Dadzie OE, Mahalingam M, Parada M, El Helou T, Philips T, et al. (2008) Adverse cutaneous reactions to soft tissue fillers--a review of the histological features. J Cutan Pathol 35: 536-548. 
Citation: Rosser JM (2014) Carbomer Viscosupplementation in the Equine Middle Carpal Joint. J Veterinar Sci Technol 5: 158. doi:10.4172/21577579.1000158

Page 6 of 6

30. Ikinci G, Senel S, Wilson CG, Sumnu M (2004) Development of a buccalbioadhesive nicotine tablet formulation for smoking cessation. Int $\mathrm{J}$ Pharm 277: 173-178.
31. Cribb NC, Bouré LP, Hanna WJ, Akens MK, Mattson SE, et al. (2009) In vitro and in vivo evaluation of ferric-hyaluronate implants for delivery of amikacinsulfate to the tarsocrural joint of horses. Vet Surg 38: 498-505. 